\title{
Coding of interceptive saccades in parietal cortex of macaque monkeys
}

\author{
Jan Churan ${ }^{1,2} \cdot$ Andre Kaminiarz $^{1,2} \cdot$ Jakob C. B. Schwenk ${ }^{1,2} \cdot$ Frank Bremmer $^{1,2}$
}

Received: 28 May 2021 / Accepted: 23 August 2021 / Published online: 1 September 2021

(c) The Author(s) 2021

\begin{abstract}
The oculomotor system can initiate remarkably accurate saccades towards moving targets (interceptive saccades) the processing of which is still under debate. The generation of these saccades requires the oculomotor centers to have information about the motion parameters of the target that then must be extrapolated to bridge the inherent processing delays. We investigated to what degree the information about motion of a saccade target is available in the lateral intra-parietal area (area LIP) of macaque monkeys for generation of accurate interceptive saccades. When a multi-layer neural network was trained based on neural discharges from area LIP around the time of saccades towards stationary targets, it was also able to predict the end points of saccades directed towards moving targets. This prediction, however, lagged behind the actual post-saccadic position of the moving target by $\sim 80 \mathrm{~ms}$ when the whole neuronal sample of 105 neurons was used. We further found that single neurons differentially code for the motion of the target. Selecting neurons with the strongest representation of target motion reduced this lag to $\sim 30 \mathrm{~ms}$ which represents the position of the moving target approximately at the onset of the interceptive saccade. We conclude that-similarly to recent findings from the Superior Colliculus (Goffart et al. J Neurophysiol 118(5):2890-2901) — there is a continuum of contributions of individual LIP neurons to the accuracy of interceptive saccades. A contribution of other gaze control centers (like the cerebellum or the frontal eye field) that further increase the saccadic accuracy is, however, likely.
\end{abstract}

Keywords Saccades $\cdot$ Visual motion $\cdot$ Parietal cortex $\cdot$ Electrophysiology

\section{Introduction}

Visual tracking of a moving object is achieved by combinations of saccades that bring the image of the object of interest onto the fovea and smooth pursuit eye movements that slowly follow a once foveated target. Saccades towards moving targets were shown to be remarkably accurate (Fuchs 1967; Cassanello et al. 2008; Fleuriet and Goffart 2012). This performance of the saccadic system is striking since it must be able to extrapolate the motion trajectory of the target to account for its own processing time before saccade onset (typically 100-300 ms) as well as for the duration of

Jan Churan

jan.churan@staff.uni-marburg.de

1 Department of Neurophysics, Philipps-Universität Marburg, Marburg, Germany

2 Center for Mind, Brain and Behavior, Philipps-Universität Marburg and Justus-Liebig-Universität Gießen, Marburg, Germany the saccade (typically $20-50 \mathrm{~ms}$ ) to accurately match the post-saccadic eye position with the position of the moving object. Which brain areas contribute to the processing and extrapolation of the target motion for purposes of interceptive saccades is still under discussion. It was previously shown that the activity of neurons in the superior colliculus (SC) does not account for the component of a saccade vector that is caused by the motion of a saccade target (Keller et al. 1996). The SC neurons in that study shifted their motion fields dependent on whether the saccades were made to stationary or moving targets in a manner that suggested that they only reflect the location of the appearance of a saccade target but not its subsequent motion. This finding gave rise to the so called 'dual-drive' hypothesis (Optican and Quaia 2002; Guan et al. 2005; Optican and Pretegiani 2017) stating that the motion information is processed in parallel and is added to the motor command at a late stage of processing probably by the caudal fastigial nuclei of the cerebellum. This notion was later refined as Goffart et al. (2017) have shown that SC neurons to a differential degree participate in 
generation of the motion-related component of interceptive saccades. Beside these findings about the role of sub-cortical areas in processing of interceptive saccades, cortical contributions along the dorsal visual pathway are little investigated yet. Lesions in the middle temporal area (MT) of macaque monkeys, which is a major center in perception of visual motion, were shown to selectively reduce the accuracy of interceptive saccades while leaving saccades to stationary targets unimpaired (Newsome et al. 1985). Target motionrelated signals were also reported in the Frontal eye field (Barborica and Ferrera 2003; Xiao et al. 2007; Ferrera and Barborica 2010) that receives input from the cortical motion areas (Tian and Lynch 1996) and has direct efferent connections to the SC (Segraves and Goldberg 1987; Stanton et al. 1988 ) and to the oculomotor areas in the brain stem (Schiller et al. 1980).

The lateral intraparietal area (LIP) is a major processing stage in the dorsal visual pathway as well as in the saccade generating circuit. Its connections make it well suited for processing of moving targets. It receives strong inputs from areas MT and the medial superior temporal area (MST, Blatt et al. 1990) that are almost exclusively engaged with processing of visual motion and sends efferent projections to the SC (Paré and Wurtz 1997) and the frontal eye field (FEF, Schall et al. 1995) which are the major sources of saccadic motor commands. Results from an earlier investigation (Bremmer et al. 2016) have shown that LIP neurons carry information about the motion of a saccade target. The goal of the current study was to specify to what degree information provided by LIP neurons can contribute to the accurate execution of interceptive saccades in $2 \mathrm{D}$ oculomotor space. To this end, we trained a multi-layer model to reproduce the trajectories of saccades to stationary targets from the peri-saccadic activity of a sample of LIP neurons. Once this model was established, we used activities from the same neurons collected during interceptive saccades as inputs to the model and tested to what degree they account for the interceptive component of the saccade trajectory, that is, the deflection of the saccade end point in the direction of target motion.

\section{Methods}

\section{Physiological preparation}

Two adult male monkeys (Macaca mulatta) participated in the study. In two separate surgeries, each monkey was implanted with a head holder and a recording chamber. Based on MRI scans prior to surgery, the chamber (inner diameter $14 \mathrm{~mm}$ ) was centered at a position $3 \mathrm{~mm}$ posterior from the inter-aural line and $15 \mathrm{~mm}$ lateral from the longitudinal fissure (P3/L15) to access the region of the intraparietal sulcus (right hemisphere in both monkeys). During the experiments, the correct position of the electrode within the lateral intraparietal area (LIP) was determined based on properties of the neurons under study as well as properties of neurons in the neighboring cortical areas, predominantly the ventral intraparietal area VIP in the depth of the intraparietal sulcus, which was identified by directional selectivity for visual motion. The correct position of the recordings was later confirmed by histology in one of the monkeys $(\mathrm{O})$. The other monkey (S) still participates in ongoing recordings. All procedures had been approved by the regional authorities and were in accordance with the published guidelines on the use of animals in research (European Communities Council Directive 2010/63/EU).

Single-unit recordings were done using standard tungsten microelectrodes (FHC, Bowdoin, USA) with an impedance of $\sim 2 \mathrm{M} \Omega$ at $1 \mathrm{kHz}$ that were positioned by a hydraulic micromanipulator (MO-95, Narishige, Tokyo, Japan). A stainless-steel guiding tube was used for transdural penetration and support of the electrode. The neuronal signal was processed using a commercial system (Alpha Omega, Nof HaGalil, Israel). It was band-pass filtered (cut-off frequencies at 500 and $8000 \mathrm{~Hz}$ ) and sampled at $44 \mathrm{kHz}$.

\section{Apparatus}

During recordings, the monkeys were sitting head-fixed in a primate chair in a dark room, and their eye position was monitored at $1000 \mathrm{~Hz}$ using a video-based eye tracker (EyeLink 1000, SR Research, Ottawa, Canada). The chair was positioned at a distance of $97 \mathrm{~cm}$ from a semi-transparent screen (size $160 \mathrm{~cm} \times 90 \mathrm{~cm}$, subtending the central $79^{\circ} \times 50^{\circ}$ of the visual field) on which the visual stimuli were back-projected using a PROPixx-projector (VPixx Technologies, St-Bruno de Montarville, Canada) running at a resolution of $1920 \times 1080$ pixels and at a frame rate of $100 \mathrm{~Hz}$.

\section{Paradigms}

The experimental paradigms were implemented using Matlab (R2012a, MathWorks, Natick, USA) and the psychophysics toolbox (Brainard 1997; Pelli 1997; Kleiner et al. 2007) on a standard Windows (v7, Microsoft, Redmond, USA) PC (Dell Precision T5810, Round Rock, USA). In all following experiments, the saccade targets were always presented on a gray background (luminance $40 \mathrm{~cd} / \mathrm{m}^{2}$ ). Before the main experiment started, the saccadic tuning of the neuron under investigation was tested during visually guided saccades to stationary targets. In this pre-test, saccades with different directions (covering the full fronto-parallel angular space of $360^{\circ}$ in steps of $45^{\circ}$ ) and different amplitudes (typically between $7^{\circ}$ and $20^{\circ}$ ) were used to find a saccade vector 
yielding strong peri-saccadic responses. This saccade-vector was then used during the main experiment, as the 'preferred vector' of the neuron. A saccade vector of the same amplitude but opposite direction ('anti-preferred vector') was also used in the main paradigm, although, the (usually very low) peri-saccadic activities from this saccade vector were only utilized to calculate the selectivity of the neuronal responses that was a criterion for a neuron's inclusion into the main analysis.

In the main paradigm, two types of trials were employed, the 'stationary' trials in which the monkeys made regular visually guided saccades towards stationary targets at different spatial positions and 'interceptive' trials where they made saccades towards targets moving in one of eight directions (Fig. 1). At the beginning of each trial, the monkey fixated a target (small red square, side length $0.8^{\circ}$ ) placed at an eccentric position on the screen for a random duration (500-700 ms). This initial position of the target was chosen so that saccades towards the locations around the screen center yielded strong peri-saccadic responses (relative to

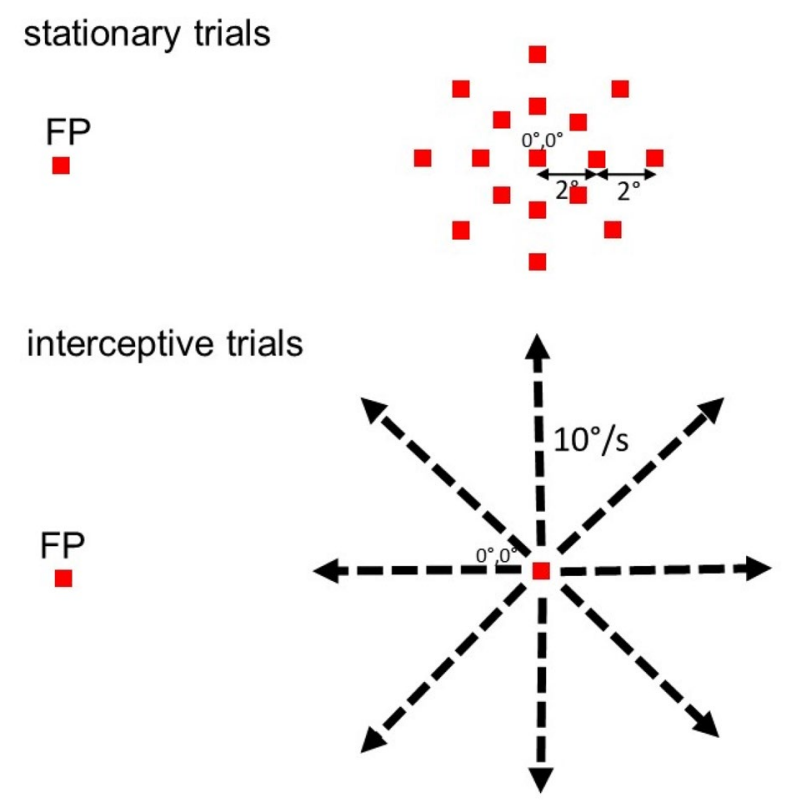

Fig. 1 Sketches of the two types of trials that were used in the main paradigm. In the 'stationary' trials (upper panel), the monkey had to make a visually guided saccade from an eccentric fixation point (FP) to one of 17 target positions that were presented around the center of the screen. The possible positions of the target are depicted on the right side of the upper panel. They included the center of the screen and positions that were $2^{\circ}$ and $4^{\circ}$ away from the center in the four cardinal and four oblique directions. These chosen positions represent locations at which the moving target in the 'interceptive' trials arrives after 200 and $400 \mathrm{~ms}$ of motion. In the 'interceptive' trials (lower panel), the monkey made a saccade from an eccentric position (which was the same as in the stationary trials) to a target that first stepped to the center of the screen and then immediately moved in one of eight directions at a constant speed of $10 \%$ the baseline activity) at least for a part of the investigated target locations. Then, in the 'stationary' trials (Fig. 1, upper panel), the fixation spot stepped to one of 17 predefined positions placed around the center of the screen and the monkey's task was to make a visually guided saccade to that position. The positions of the target were chosen in the center of the screen $\left(0^{\circ} / 0^{\circ}\right)$ and along the cardinal as well as the oblique axes in distances of $2^{\circ}$ and $4^{\circ}$ from the screen center. These specific positions were chosen to be identical with the positions of the moving targets in the interceptive trials after $200 \mathrm{~ms}$ and after $400 \mathrm{~ms}$ of motion and hence to be close to the average landing points of the interceptive saccades. The saccade had to be initiated within $300 \mathrm{~ms}$ from the stimulus step and land within an $8^{\circ} \times 8^{\circ}$ window around the target. After the saccade, the monkey had to fixate the target for another $500 \mathrm{~ms}$ to obtain a liquid reward. Typically 10 trials were collected for each of the 17 different target positions. 'Interceptive' trials (Fig. 1, lower panel) started with fixation of a stationary target at the same eccentric position as in the 'stationary trails'. The monkey then made a saccade towards a target that first jumped to the center of the screen and then immediately started moving into one of 8 directions at a speed of $10 \%$ s. Again, the saccade had to occur within $300 \mathrm{~ms}$ after the step of the target and had to land within an $8^{\circ} \times 8^{\circ}$ window around the current target position. After the saccade the monkey had to follow the target using smooth pursuit eye movements (SPEM) for another $800 \mathrm{~ms}$ to receive a liquid reward. Typically, 15 trials were collected for each direction of stimulus motion. Both, the stationary and the interceptive trials were presented randomly interleaved during a single measurement. In the following, we will name the saccades to the stationary targets 'regular saccades' and those to moving targets 'interceptive saccades'.

\section{Data processing and analysis}

\section{Processing of eye movements}

The eye position signal was smoothed by a moving average filter with a span of $15 \mathrm{~ms}$. Then, the initial saccades were detected using a double velocity criterion in which at first time-windows were detected at which the eye velocity exceeded $100 \% \mathrm{~s}$ and then in a second step, the beginning and the end of a saccade were identified when the velocity before and after these time-windows first fell below $30 \%$ s. The pre-saccadic and the post-saccadic eye positions were calculated as averages in a time window $6-16 \mathrm{~ms}$ before the start and 2-4 ms after the end of the saccade. The very short post-saccadic time window was chosen to avoid a contamination by the subsequent SPEM in the interceptive trials. The results were confirmed visually for a subset of the 
trials to give accurate estimates of pre- and post-saccadic eye positions.

\section{Processing of neuronal data}

Single units were isolated using a semi-manual spike sorter (Plexon Inc, Dallas, Texas). To this end, we used a threshold on the electrode signal that was set manually to separate the action potentials from noise. The samples that exceeded the threshold were further analyzed using principal components as well as other features that were derived from the signal (like local maxima and minima). Then, clusters of samples with similar properties were identified visually and grouped together as a single unit. For a detailed description of the sorting process, see the offline User Guide (Plexon 2020). The procedures used for further analysis of the data were written in MATLAB (R2012a, MathWorks, Natick, USA),

\section{Generation of a pseudo-population}

The data basis for this investigation consists of the activity of a sample of 105 neurons ( 50 for monkey S, 55 for monkey O) that were tested using both paradigms as described above. All neurons have shown a significant difference in activity $(p<0.01, t$ test) between the preferred and the anti-preferred saccade direction in a time window between $100 \mathrm{~ms}$ before and $100 \mathrm{~ms}$ after the saccade onset.

Separate datasets were required for training and validation of the neural network model. Thus, the data from the stationary trials were divided into a training sample that consisted of $70 \%$ of the trials and a validation sample that consisted of the remaining 30\%. The processing steps described below were performed separately for the training and the validation trials. To minimize random effects caused by splitting of the datasets, we performed this procedure 50 times resulting in 50 training datasets and corresponding 50 validation datasets.

It is important to note that different neurons within the sample were recorded during separate sessions and the preferred saccade directions and amplitudes could differ between the measurements dependent on the properties of the investigated neuron (the distribution of all investigated saccade directions during all measurements as well as the distribution of all preferred saccade directions are shown in Figure S-1). Consequently, the recorded samples cannot be used to decode the full vector of the saccades but only their end positions which were always similar between the recordings. To combine the activities of all neurons to create the model, we first had to align their activity profiles to the same spatial and temporal coordinates. The procedure is illustrated in Fig. 2a, b. We first calculated continuous peri-saccadic response functions for each target position in the stationary paradigm by convolving the spike times (relative to the saccade onset) with a Gaussian $(\sigma=20 \mathrm{~ms})$, and averaging over all collected trials. Then, we interpolated the neuronal activities for $\mathrm{x} / \mathrm{y}$ positions in between the measured saccade end-positions using linear interpolation. When an extrapolation of the activities was necessary to obtain a tuning over the same spatial area for each neuron, we chose the next-neighbor extrapolation method to not overestimate the activity outside of the investigated area. The resulting data matrix for each neuron sampled the area around the center of the screen from $-4^{\circ}$ to $+4^{\circ}$ in $0.1^{\circ}$ steps for the horizontal and the vertical dimensions and the peri-saccadic times from $400 \mathrm{~ms}$ before the saccade onset to $350 \mathrm{~ms}$ after saccade onset in steps of $10 \mathrm{~ms}$.

The same procedure was used for estimating the spatiotemporal activity profiles during the interceptive trials, using 8 saccade end-positions that resulted from the different directions of target motion. Thus, the spatial area in which the interceptive saccades were investigated was smaller than for regular saccades since the saccade end points were deflected by the stimulus motion on average only by $\sim 1.5^{\circ}$ (see Fig. 3) so the relevant area was only $\sim 1.5^{\circ}$ in each direction around the center of the screen.

\section{Training and validation of the neural network models}

For large part of the investigation, we used a rather broad time-window between $100 \mathrm{~ms}$ before and $100 \mathrm{~ms}$ after the onset of the saccade - the times at which most of the saccade-related activity takes place. To investigate the time course of saccadic information, we also used time-windows of $100 \mathrm{~ms}$ duration that were shifted in steps of $50 \mathrm{~ms}$ in the range between $400 \mathrm{~ms}$ before and $350 \mathrm{~ms}$ after the saccade onset. For the training of the networks, a large training sample is advantageous to avoid overfitting of the data by the model. Thus, we generated a large set of trials based on the estimated spatio-temporal activity profile of each neuron (Fig. 2c) and the assumption that the spike counts in different trials are approximately Poisson distributed (e.g., Shadlen and Newsome 1998). To this end, we chose a random saccade end-position between $-4^{\circ}$ and $+4^{\circ}$ in horizontal and vertical directions (white circles in Fig. 2c) and calculated the mean expected spike count within the investigated time window for each neuron, based on the training sample of the recorded trials. Then, we used this average spike count as the estimate for the mean of a Poisson distribution from which the actual trial was randomly drawn. This procedure was repeated 100.000 times to sample well the whole investigated spatial area.

For a validation of the model, we either generated a similar dataset based on the validation trials or, for better clarity of presentation, we investigated the model predictions at discrete saccade end locations. To allow for comparison between the predictions of the model for regular and 


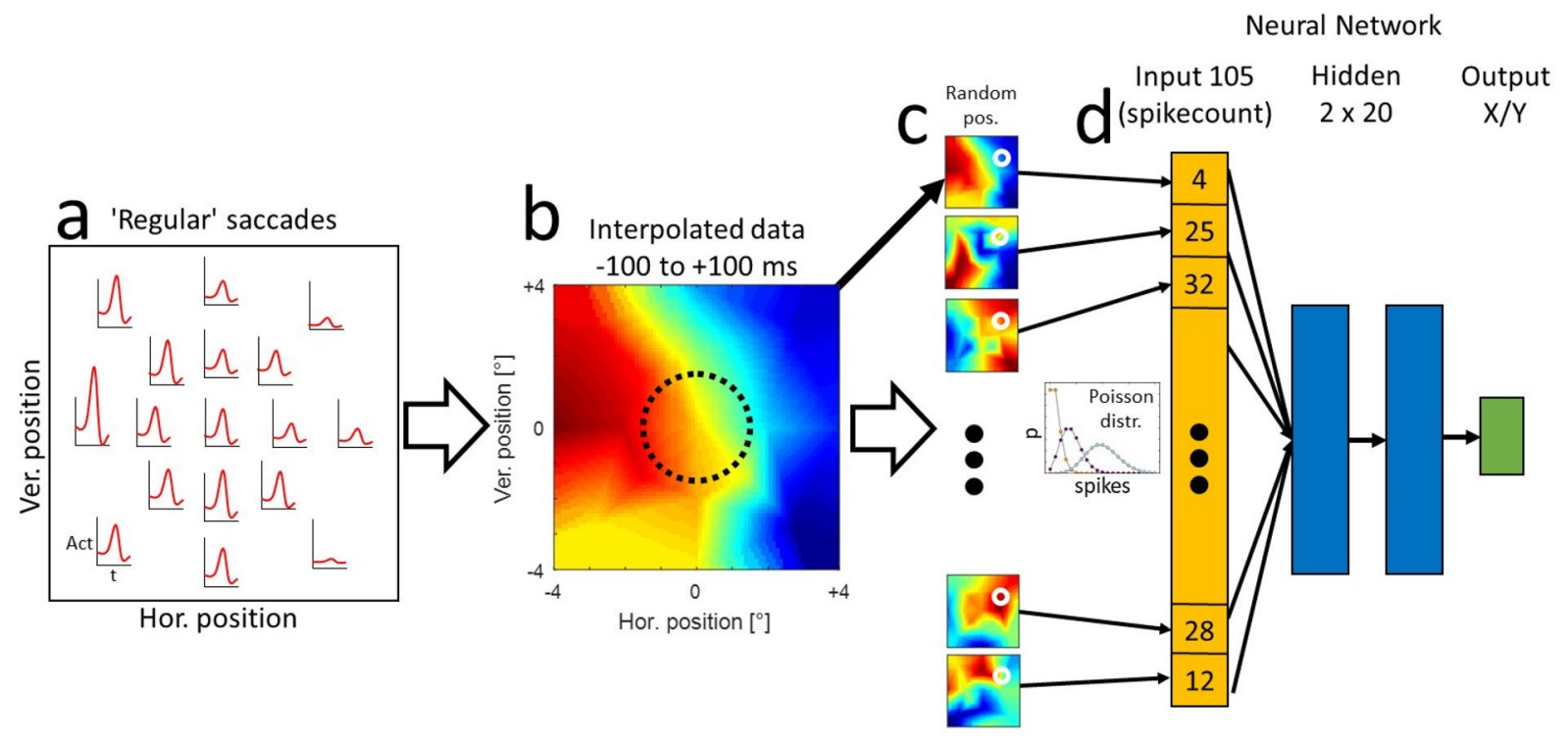

Fig. 2 Processing steps towards a model predicting the end-position of regular saccades from activity of the sample of 105 neurons. a For one example neuron, the peri-saccadic activity was sampled from 17 positions around the center of the screen. $\mathbf{b}$ The activities were then interpolated over space and time in the range of $\pm 4^{\circ}$ in horizontal and vertical directions. The average activity in a time window of $\pm 100 \mathrm{~ms}$ around the saccade onset was used for the training of the neural networks. The dotted circle represents a smaller area in which the interceptive saccades and regular saccades were later compared.

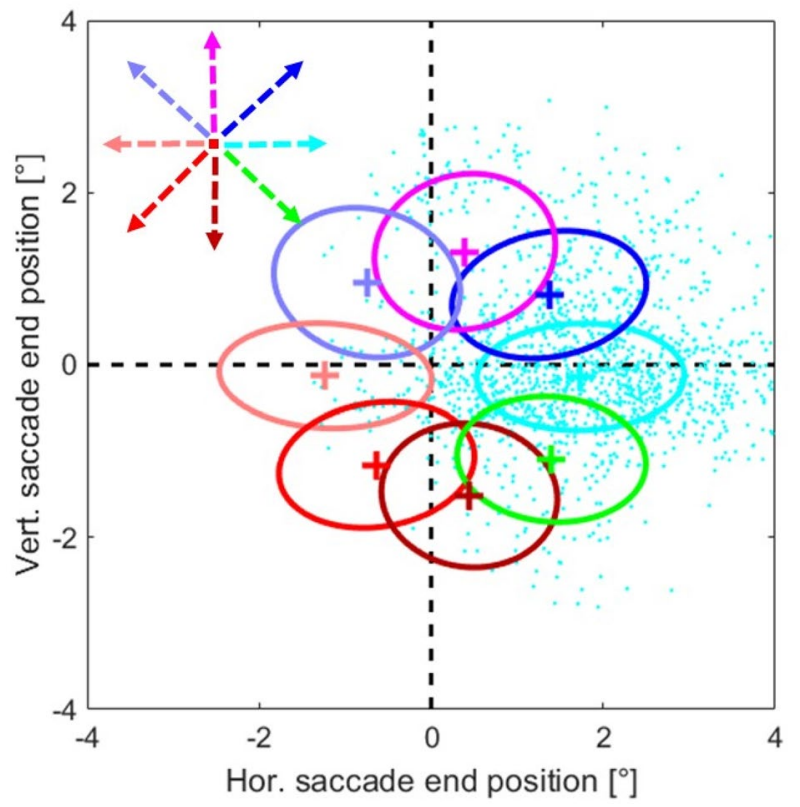

Fig. 3 Two-dimensional Gaussians fitted to the end-positions of interceptive saccades made by both monkeys during all recording sessions. Different colors represent different target directions, crosses show the mean saccade end-position and the borders of the ellipses represent one standard deviation around the mean. Raw data depicting the end points of the saccades (cyan dots) are only shown for target movement to the right c For each training dataset, a random spatial position was chosen and the activity of each of the investigated neurons at that position (white circles) was used as a mean of a Poisson distribution from which the number of spikes in each trial was randomly drawn. $\mathbf{d}$ The randomly generated trials were used to train the neural network model consisting of 105 elements in the input layer and 2 hidden layers with 20 elements each to predict the horizontal and vertical coordinate of the landing position of the saccade

interceptive saccades, we have chosen these discrete locations as the average end positions of interceptive saccades for different directions of target motion (Fig. 3). In this way, the model was tested only at those spatial positions where sufficient data were obtained for regular as well as for interceptive saccades.

For the decoding of the saccade end points from the activities in our neuronal sample, we chose a shallow neural network model as implemented in the MATLAB Neural Network toolbox (R2018a). In our model, the size of the input layer was determined by the size of the neuronal sample (105) and the size of the output layer by the content of the desired output (x-position and y-position, 2 elements). For the hidden layers, we have tested several configurations which did not show large differences regarding the accuracy of the predictions and chose two hidden layers with twenty elements each (Fig. 2d), which have shown a good accuracy as well as a relatively fast training speed. The networks were trained using the Levenberg-Marquardt algorithm (Levenberg 1944; Marquardt 1963). A separate network was trained for each of the 50 splits between the training and the validation samples and for each investigated time window. After the training, the performance of the networks to predict (1) saccade end-positions of regular saccades and (2) saccade end-positions of interceptive saccades was tested. 
As mentioned above, for regular saccades, the separately generated validation samples were used for this testing. In contrast, for the study of interceptive saccades, no splitting of the data was necessary since the trials from interceptive saccades were always independent from the training trials and thus here the full data sample was used.

\section{Results}

\section{Interceptive saccades}

During the single unit recordings, we collected the data from altogether 11,631 interceptive saccades, including saccades in the preferred as well as the anti-preferred directions of each neuron (for the distribution of the preferred saccade directions over all measurements see Figure S-1). For a brief overview-the average amplitude of these saccades was $9.7^{\circ}\left(\operatorname{std} 3.9^{\circ}\right)$, the average latency was $134 \mathrm{~ms}$ ( $\operatorname{std} 44 \mathrm{~ms}$ ) and the mean duration (time between the onset of the saccade and its end) was $36 \mathrm{~ms}$ (std $8.9 \mathrm{~ms}$ ). To confirm the expected effect of target motion on saccadic eye movements, we investigated the end points of saccades towards targets moving from the center of the screen (coordinate $0^{\circ} / 0^{\circ}$ ) in eight different directions. We fitted 2D Gaussians to the saccade end-positions separately for each motion direction as shown in Fig. 3. The results demonstrate that the motion of the stimulus exhibited a clear influence on the end-positions

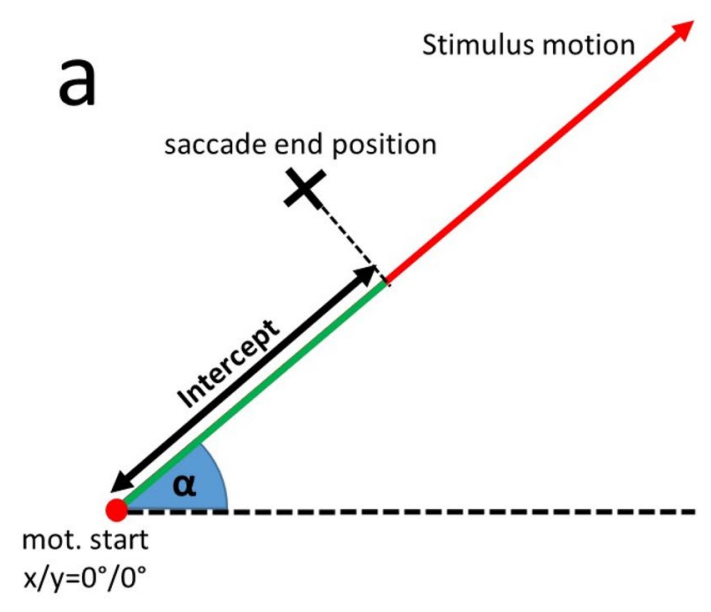

Fig. 4 a Description of the 'Intercept' as the component of saccade end-position that was aligned with the motion vector of the target. $\mathbf{b}$ Relationship between the timing of the end of the interceptive saccades and their intercepts. The data from 11,631 interceptive saccades from both monkeys are shown as green crosses, the correlation between the time of saccade end and the intercept was 0.39 of the interceptive saccades. They were, on average, shifted in the direction of the target motion, however, there was a major spread of the end-positions as can be seen by the example data points (cyan) that are provided for one-motion direction. Another hallmark of interceptive saccades is the dependence of their trajectory on their timing (Quinet and Goffart 2015; Bremmer et al. 2016), in that the target-motion-dependent component becomes larger at longer saccade latencies. This dependence is confirmed in Fig. $4 \mathrm{~b}$ that shows the relationship between the time of the saccade end and the motionrelated component of the saccade. For that purpose, the saccade end-position is expressed as the 'Intercept' - which is the orthogonal projection of the saccade end-position on the vector of the target motion (Fig. $4 \mathrm{a}$ ) and was calculated as:

intercept $=x * \cos (\alpha)+y * \sin (\alpha)$

where $x$ and $y$ are the coordinates of the saccade end-position and $\alpha$ is the angle representing the direction of target motion.

Although the landing positions in single trials have shown some variability (std of regression residuals $=0.82^{\circ}$ ), they show a clear correlation between the time of saccade end and the intercept (Pearson correlation: $r=0.39, p<0.001)$. The regression line fitted to the data (black line) resembled the target position at the end of the saccade (gray line) which is also supported by the resulting equation of the regression in which the slope was $9.6 \%$ (target speed was $10 \%$ s) and the crossing of the $\mathrm{y}$-axis was at $-0.17^{\circ}$. All target directions were collapsed

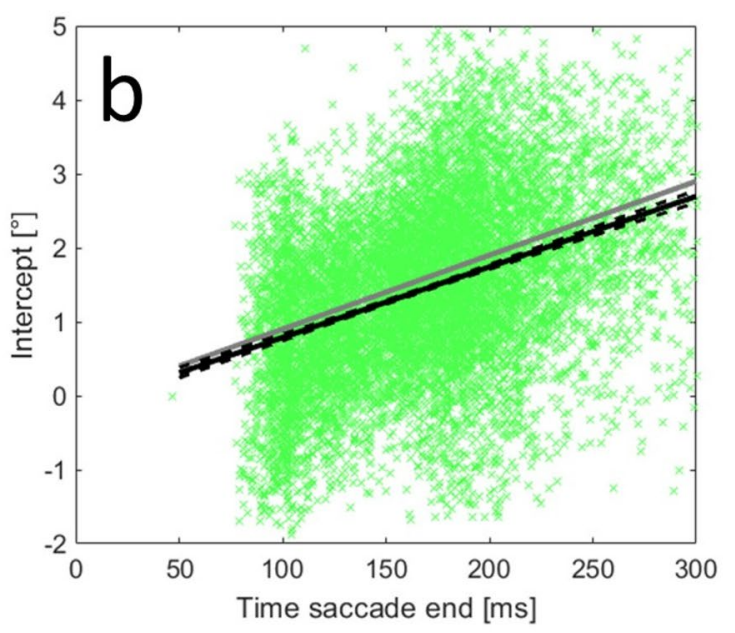

$(p<0.001)$. The gray line marks the position of the target (relative to the center of the screen) at the respective time. The black line shows the results of a linear regression of the data and the black dashed lines its confidence interval ( $p=0.01$, as calculated from a bootstrapping procedure). The motion directions of the targets were collapsed for this analysis, results for individual directions are shown in Figure S-2 
for this analysis, results for individual directions are shown in Figure S-2.

\section{Single-unit responses}

As described in the methods section, we interpolated the activities of single neurons recorded during saccades towards stationary targets as well as during interceptive saccades between different spatial positions and different times relative to the saccade onset. We focused on the area of $4 \times 4$ degrees around the center of the screen since this is the area where most of the interceptive saccades landed. The time course of the peri-saccadic activity in area LIP was in detail described elsewhere (e.g., Barash et al. 1991). Thus, in Fig. 5, we only show the temporal profiles at different spatial positions for one example neuron. The profiles show two typical features that were found in most of the neurons in the investigated sample. First, the neurons reached their maximal activity around the time of saccade onset and, second, they show some degree of saccade-related activation at all investigated spatial locations, however, the amount of activation varied between locations.

Next, we compared the activity profiles obtained from the regular and the interceptive saccades. The activity maps at the time of the saccade onset from four example neurons are shown in Fig. 6 (more examples from another four neurons are shown in Figure S-3). From a visual inspection, activity profiles from two neurons shown in the left two columns appear to show a good resemblance for regular and interceptive saccades while profiles from two other neurons shown in the right columns show no such resemblance. We used two quantitative measures to calculate the degree of similarity between the profiles. A Pearson correlation between the profiles which was 0.75 and 0.87 for the neurons in the left columns and 0.12 and -0.17 for the neurons in the right columns. We also calculated a similarity index (Sotero et al. 2010) as:

$\mathrm{SI}=1-\frac{\|z(R)-z(I)\|_{\mathrm{F}}}{\|z(R)\|_{\mathrm{F}}+\|z(I)\|_{\mathrm{F}}}$

where $\|X\|_{\mathrm{F}}$ is the Frobenius norm of $X, z(R)$ is the z-transformed activity profile for regular saccades and $z(I)$ is the $z$-transformed activity profile for interceptive saccades. The value of SI is always between 0 and 1 , where 1 would be only achieved when $\mathrm{R}$ and I were identical. In the examples in Fig. 6, the SIs of the neurons in the left column were 0.43 and 0.70 and in the right column 0.12 and 0.16 . For more robust results, we next averaged the activity in the time window between $100 \mathrm{~ms}$ before and $100 \mathrm{~ms}$ after the saccade onset and used these maps to compare the activity profiles for all neurons in our sample. The results are shown in Fig. 7. The correlations and the SI values point towards a continuum in degrees of similarity between the activity profiles for the two types of saccades. At least in a part of the sample of neurons, saccade-related tuning appears to be very similar, independent of whether the saccade endposition was systematically changed by different positions
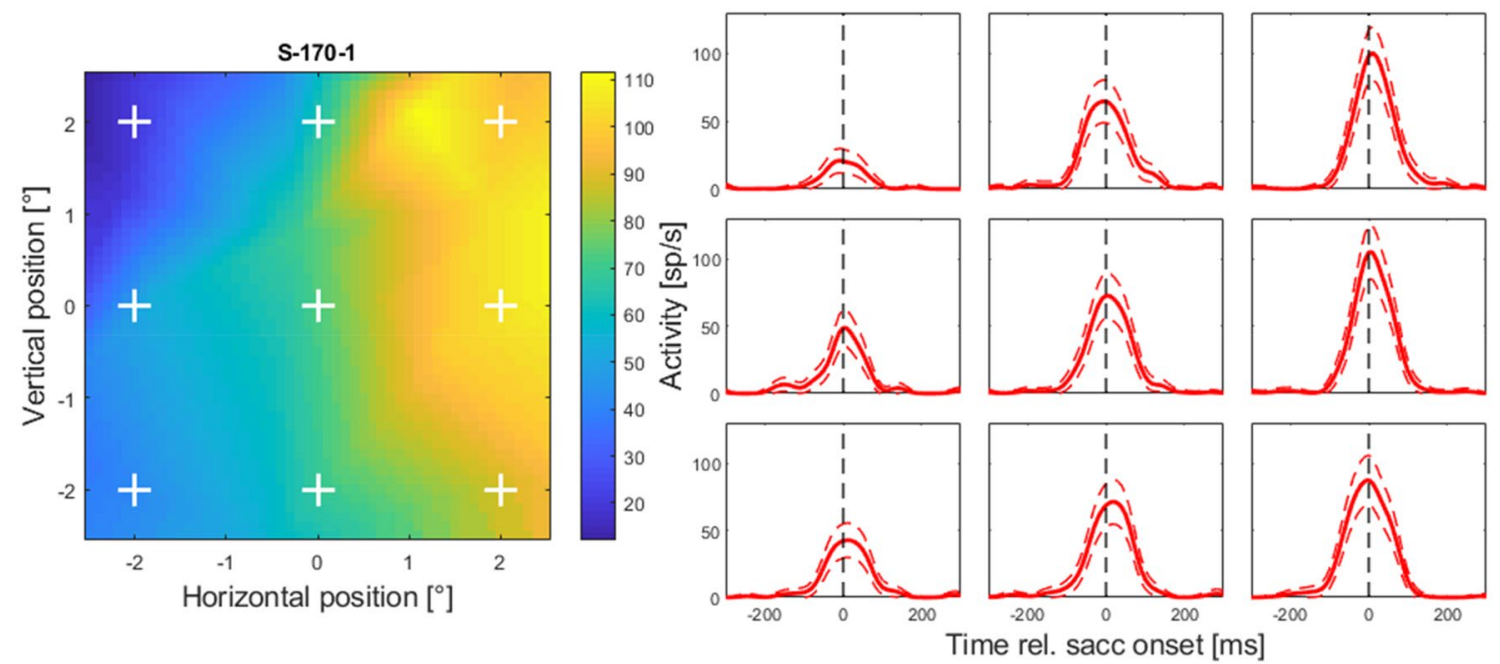

Fig. 5 left: Spatial activity profile of one example neuron at saccade onset (initial fixation was at $x=0^{\circ}, y=7^{\circ}$ ). The profile was calculated using the spatial and temporal interpolations as described in "Methods". White crosses are marking the positions from which the temporal profiles are shown on the right, the arrangement of crosses in the left corresponds with the plots on the right. Right: Time course (mean

and $95 \%$ confidence interval) of the interpolated peri-saccadic activity taken at nine example positions around the center of the screen. Note, that the example positions were chosen to give an overview over the investigated spatial area and do not coincide with the locations of the saccade targets used in the experiment 


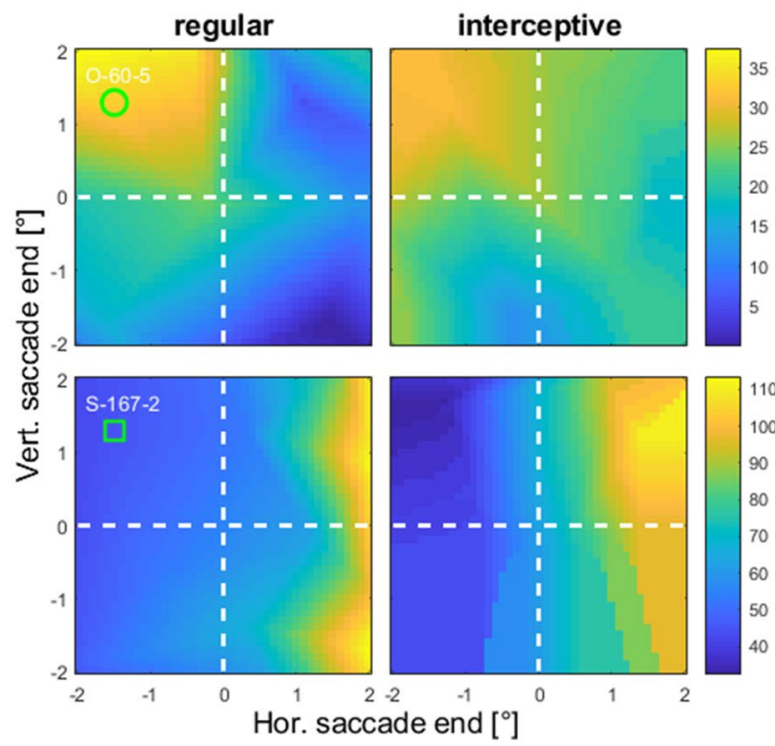

Fig. 6 Spatial tuning profiles from four example neurons calculated from a Gaussian window $(\sigma=20 \mathrm{~ms})$ centered on saccade onset for regular and interceptive saccades. The positions at the $x$ and $y$ axes represent the locations of saccade endpoints relative to the center of the screen. The two examples in the left two columns show an apparent similarity between the two activity profiles while for the neurons whose activity is shown in the two columns on the right no such simi-

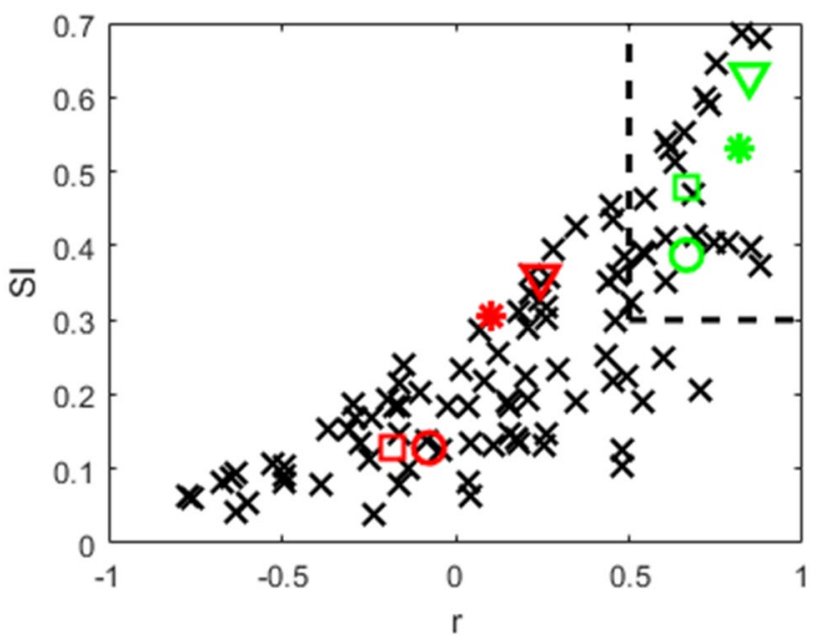

Fig. 7 Measures of similarity between the activity profiles for saccades to stationary targets and interceptive saccades for a sample of 105 LIP neurons. The activity profiles were averaged in a timewindow between $100 \mathrm{~ms}$ before and $100 \mathrm{~ms}$ after saccade onset. The Pearson correlation coefficients are plotted at the $\mathrm{x}$-axis and the similarity coefficients (as defined in Eq. 1) on the y-axis. The red and the green markers show example neurons from Figs. 6 and S-3. Dashed lines mark the area containing 25 neurons that were used in a later analysis of neurons with highest similarity of profiles between regular and interceptive saccades

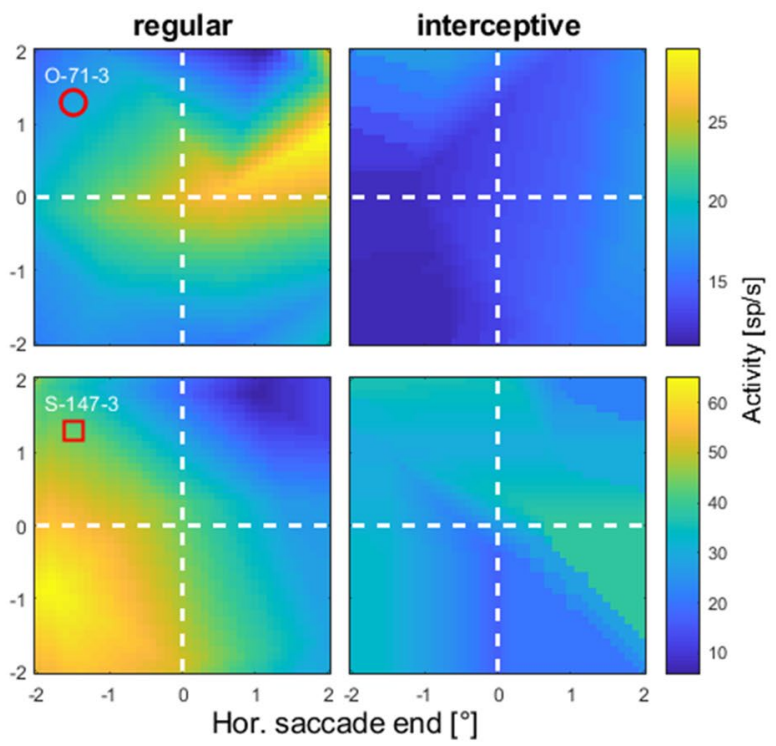

larity was observed. Red and green markers below the cell codes correspond to the positions of each example neuron in the distribution of similarity indices shown in Fig. 7. Note that the color scales were chosen rather to optimally compare the tuning profiles within single neurons than to make comparison between the neurons. Data from another four example neurons are shown in Figure S-3

of stationary saccade targets or by interception of targets moving in different directions. Note also that some neurons show a high correlation between the two profiles paired with a low SI value. In these neurons, the overall tuning pattern of activities was similar (as indicated by high positive correlation coefficient) but the average activity rate was very different between regular and interceptive saccades.

\section{Representation of regular and interceptive saccades in LIP population activity}

We trained neural network models to predict the landing points of saccades towards stationary targets from the activities of our sample of 105 LIP neurons. As described in the Methods, we split the data in $70 \%$ training and 30\% validation trials 50 times to mitigate the effects of random differences between the splits. Figure $\mathrm{S}-4 \mathrm{a}$ shows an example of a validation test of one of the resulting 50 networks that was trained on activity in the time-window between -100 and $+100 \mathrm{~ms}$ relative to the saccade onset. As stated above, we decided to test the performance of the model at locations that represented the average landing points of interceptive saccades because here sufficient data were collected for the regular as well as for the interceptive saccades and thus a fair comparison of the two was possible. Figure S-4a shows the model results for the validation trials from one of the networks for regular saccades. The averages of the estimates 
apparently fit the tested positions well in most cases. This is also supported by the confusion matrix on the right side of the plot in which the trials are assigned to one of the eight tested positions based on the minimal Euclidian distance. On average, $47 \%$ of single trials were assigned correctly (12.5\% are expected by chance), and on average, $88 \%$ were assigned correctly or to one of the direct neighbors (37.5\% are expected by chance). Some of the tested positions (e.g., position 3) show a systematic error of the predictions which likely is a result of random effects of splitting the original data into training and validation samples. In Figure S- $4 b$, the same neural network as in S-3a was tested using data from interceptive saccades. Also, here the distributions of predicted saccade end-positions deviate from the center towards the tested positions. This is also confirmed by the confusion matrix showing on average $36 \%$ of correct assignments and $75 \%$ of assignments that were either correct or one of the direct neighbors. In this case, however, a clear bias towards the center of the screen can be observed for all tested positions.

We combined the predictions for all 50 networks that were trained using the different data splits. The results are shown in Fig. 8. Here, the mean predicted positions from all tested networks are marked as a cross and the averaged standard deviations for all tested networks in $\mathrm{x}$ and $\mathrm{y}$ directions are shown as an ellipse. As in Figure S-4, we found that the predicted end-positions of regular saccades (Fig. 8a) were on average quite accurate with a mean error of $0.38^{\circ}$ (for comparison-the typical accuracy of the EyeLink eyetracker is reported to be $\left.0.25^{\circ}-0.5^{\circ}\right)$. The confusion matrix showed on average $37 \%$ correct assignments and $81 \%$ correct or direct neighbor assignments. We also found that the neural networks were capable to predict saccade end-positions of interceptive saccades (Fig. 8b); here, the confusion matrix showed $34 \%$ of correct assignments and $74 \%$ correct or direct neighbor assignments. However, a systematic error towards the center was also observed for all tested positions. For an easier comparison between the two types of saccades, we calculated the 'center bias' of the predictions as the difference between the intercept (Fig. 4a, Eq. 1) of the tested position and the intercept of the prediction. A positive value indicates that the predicted saccade position is shifted towards the center relative to the tested position and vice versa. In Fig. 8c, d, the center bias is shown for the different tested positions and as averages over all positions. The center bias for interceptive saccades was on average $0.75^{\circ}$ and significantly ( $p<0.01$, paired t test) higher than for regular saccades (average $0.25^{\circ}$ ).

We further investigated this systematic pattern of prediction errors by making the model networks predict saccade end points in a continuous area in a range of $1.5^{\circ}$ around the center of the screen (coordinate 0/0). Figure $9 a$, b shows the error vectors between the tested position (arrow backs) and the prediction of the model (arrow tips) for regular (a) and interceptive (b) saccades. Note that in this figure, for a better overview, we have chosen to reduce the number of presented locations and reduced the length of the arrows (representing the size of the error) by $50 \%$. The figures show that while there is no clear pattern of errors for the regular saccades, the predictions for the interceptive saccades are clearly biased towards the center. This center bias increases with increasing eccentricity of the tested positions. The details about the direction and the amplitude of the errors for all tested positions are then shown in Fig. $9 \mathrm{c}-\mathrm{f}$ for all tested positions. They confirm that while for the regular saccades only small (albeit systematic) errors were observed (c,e), for the errors for interceptive saccades a pinwheel structure of error directions (d) indicates a compression of the predictions towards the center of the screen. The strength of the error grows with increasing eccentricity with the exception of an area in the upper right quadrant in which the predictions were more accurate than in the rest of the tested positions (f).

Results shown in Fig. 7 indicate, that there is a continuum of similarities between the activity profiles between regular and interceptive saccades in the LIP neuronal population, ranging from very different to close to identical. Thus, we asked whether these similarities also reflect the ability of the neurons to code for the end-position of interceptive saccades (and consequently represent the movement of the target). To test this, we focused on a sub-sample of neurons with the strongest similarity between the two activity profiles as obtained from the regular and interceptive saccades. For this sample of 'best' neurons, we selected 25 neurons in which the Pearson correlation between the spatial activity profiles for regular and interceptive saccades was larger than 0.5 and the Similarity index was larger than 0.3. These borders are marked by a dashed line in Fig. 7. Using this sample of neurons, we again trained the neural networks for predicting the endpoints of interceptive saccades based on training data taken from regular saccades. The results are shown in Fig. 10 in the same format as Fig. 8. It indicates that while the predictions were on average accurate for regular saccades, the center bias that was previously observed for interceptive saccades was not fully eliminated albeit strongly diminished and was now on average only $0.3^{\circ}$. Given the target speed of $10 \%$, this spatial bias can be transformed into a $30 \mathrm{~ms}$ time-lag on the target, which corresponds well with the position of the target at the beginning of the interceptive saccade (average duration of interceptive saccades was $36 \mathrm{~ms}$ ).

\section{Time course of motion information processing}

Up to this point, we investigated the activity collected in a rather broad time-window between $100 \mathrm{~ms}$ before and $100 \mathrm{~ms}$ after the saccade onset. To obtain a temporal profile 

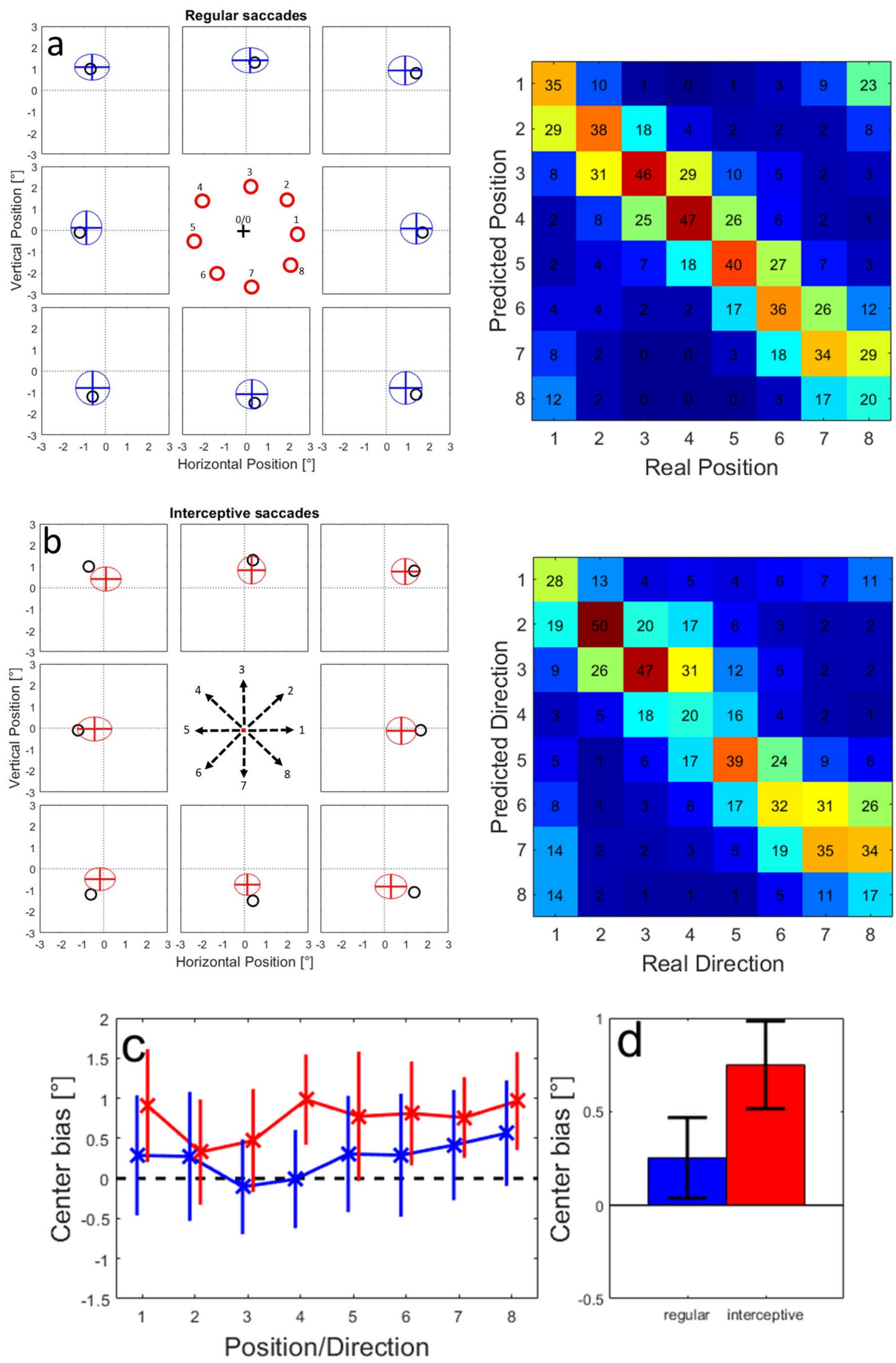
4Fig. 8 a Validation of the training success for 50 networks that were trained on $70 \%$ of the available data from regular saccades and tested using the remaining $30 \%$. Black circles show the tested positions, the ellipses represent the average positions and average standard deviations from 50 validation samples. In the confusion matrix on the right, the predictions from single trials over all validation samples were assigned to one of the tested positions based on the minimal Euclidian distance between the prediction and each of the tested positions. The numbers indicate the percentages of assignments for each tested ('real') position. b The same plot for predicting the endpositions of interceptive saccades from the population activity based on neural network models trained using data from regular saccades. c Averages and standard deviation of the Center bias for different tested positions for regular (blue line) and interceptive (red line) saccades. The lines are slightly shifted for better visibility. d Averages and standard deviations of the center bias for regular (blue) and interceptive (red) saccades calculated over all tested positions

of the saccade-related information in our neuronal sample, we also trained neural networks based on activity in timewindows of $100 \mathrm{~ms}$ duration that were moved in steps of $50 \mathrm{~ms}$ starting from $400 \mathrm{~ms}$ before and ending at $350 \mathrm{~ms}$ after the saccade onset. As described in the Methods, the data in each time-window were again split fifty times into training and validation datasets and the networks were trained on the training datasets of each of these splits separately. We used the same example saccade end-positions as in previous sections to evaluate the predictions of the model for regular and interceptive saccades. The supplemental videos S-5 and S-6 show the development of the means and the standard deviations of the predictions over time in the same format as in Fig. 8. The line intersections represent the average predicted saccade end-position for regular (video S-5) and interceptive (video S-6) saccades. The ellipses indicate the average standard deviations of the predictions in horizontal and vertical directions. Two effects can be observed: (1) In a narrow time-window around the onset of the saccade, the average predicted positions move from the center in the direction of the tested positions. (2) The variability of the predictions (indicated by the radii of the ellipses) decreases in approximately the same time-window. The two effects are summarized in Fig. 11a, b for regular (blue) and interceptive (red) saccades. For regular saccades, it shows a decrease of the center bias starting at $-100 \mathrm{~ms}$ and reaching a minimum at saccade onset. For interceptive saccades, the temporal profile appears shifted by $\sim 50 \mathrm{~ms}$, the center bias starts decreasing $50 \mathrm{~ms}$ before and reaches a minimum $50 \mathrm{~ms}$ after the saccade onset. Consequently, the largest differences between the predictions for regular and interceptive saccades were observed before and at saccade onset. The time-windows in which the center bias for the two saccade types was significantly different (paired $t$ test, $p<0.01)$ are marked by asterisks. After the saccade, center bias increases again for both types of saccades as the predictions move back towards the center. No significant differences were observed in the post-saccadic time period. When investigating the trial-by-trial variability of the predictions (Fig. 7b), an increase of precision (as marked by a decrease in the variability of the predictions) can be observed around saccade onset. No significant differences between the saccade types were found except for a brief period $200 \mathrm{~ms}$ after saccade onset to which we do not assign any specific meaning. Next, we used the same procedure on the subpopulation of 25 neurons that have shown the highest similarity of tuning profiles between regular and interceptive saccades as described above. The results are shown in supplemental videos S-7 and S-8 and summarized in Fig. 11c, d. For the center bias (Fig. 11c), similar differences between the temporal profiles for the two saccade types are shown as in the whole sample of neurons (Fig. 11a). Also here, the presaccadic coding of the saccade end-position for interceptive saccades is delayed by $\sim 50 \mathrm{~ms}$ relative to regular saccades but now having the smallest bias at the same time, i.e., saccade onset. While there was a substantial center bias, for the predictions of interceptive saccades based on the whole neuronal sample, this bias was close to zero at the saccade onset when the model was only based on the selected sample of 25 neurons. The temporal profile of the variability of the predictions (Fig. 11d) was less pronounced and has shown a larger post-saccadic variability for the predictions of interceptive saccades. This might be caused by the behavioral variability induced by the smooth pursuit eye movements (including catch-up saccades) that followed the interceptive saccade in contrast to a steady fixation that was following the regular saccades.

\section{Discussion}

\section{Precision and accuracy of interceptive saccades}

The analysis of saccade end-positions shown in Figs. 3 and 4 indicates that the saccade trajectory was clearly shifted in the motion direction of the eye-movement target. On average, we found the landing position of the saccades to lag $0.25^{\circ}$ behind the target which was well in line with previous findings (Gellman and Car 1991). The average Euclidean error (calculated as the Euclidean distance between the saccade end point and the target position) in our measurements was $1.48^{\circ}$ which was slightly higher than reported by Goettker et al. (2019) for human subjects where it was on average $\sim 1.1^{\circ}$. We observed a similarly reduced precision of oculomotor performance in monkeys relative to human observers in an earlier study on eye movements towards moving targets (Churan et al. 2018). We do not believe that these differences reflect a generally lower ability of macaques to perform precise and accurate eye movements but are rather mediated by the different conditions during data acquisition. While human subjects in general perform 


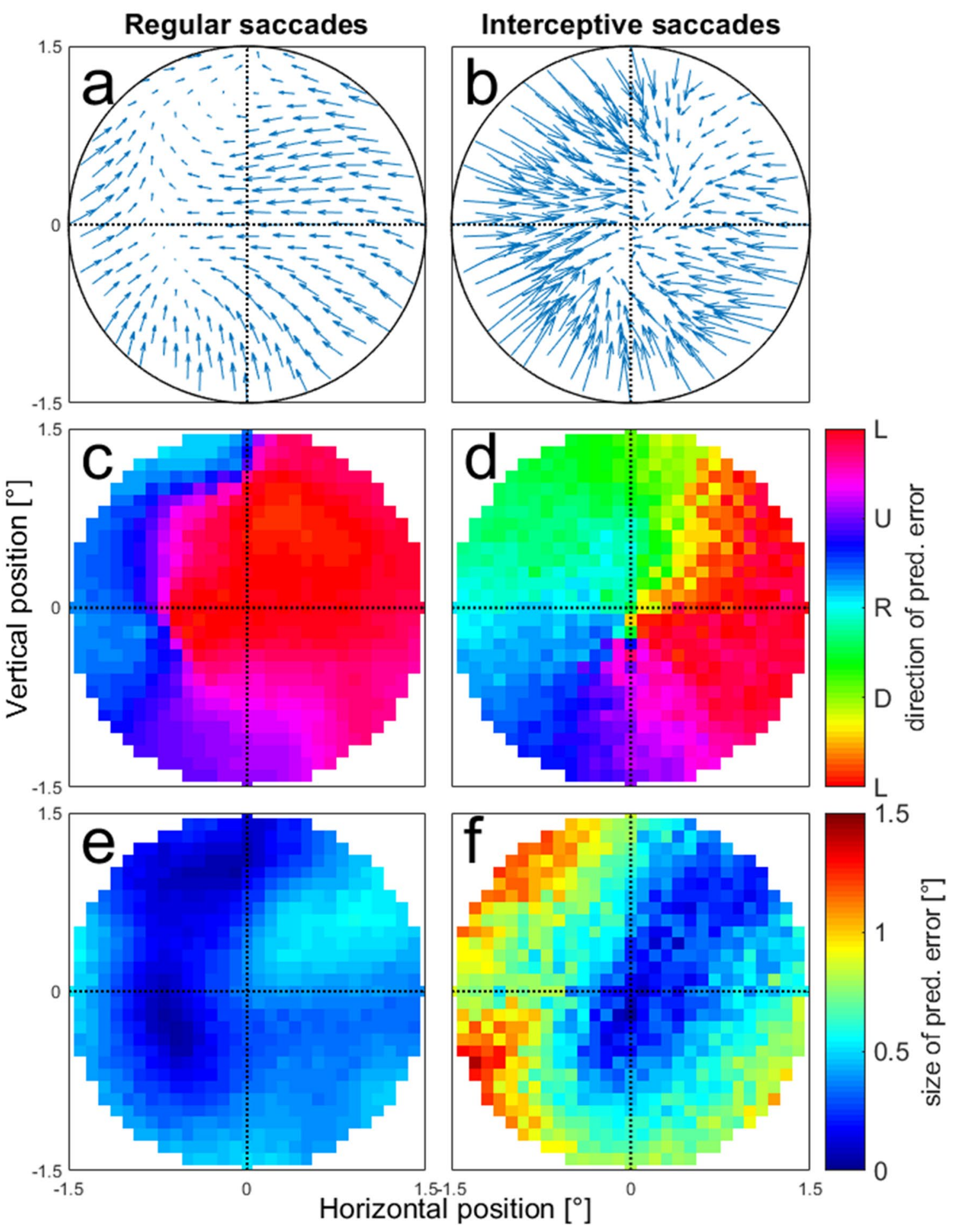

Fig. 9 Prediction errors of saccade end-positions tested at different positions in the area of $1.5^{\circ}$ around the screen center. a, b Average error vectors shown at selected positions. The arrows indicate the differences between the tested positions and the predictions of the model. The length of the error vectors is scaled down by $50 \%$ for a better overview. c, $\mathbf{d}$ Average directions of the error vectors for regular (c) and interceptive (d) saccades. e, f Average length of the error vectors for regular (e) and interceptive (f) saccades the task for a very limited duration and enjoy frequent breaks, the recordings require a continuous performance of several hundred of trials, which is repeated over many days.
Thus, it is likely, that under these conditions, the training on the specific task, but also the allocation of attentional resources and general vigilance are different between human 

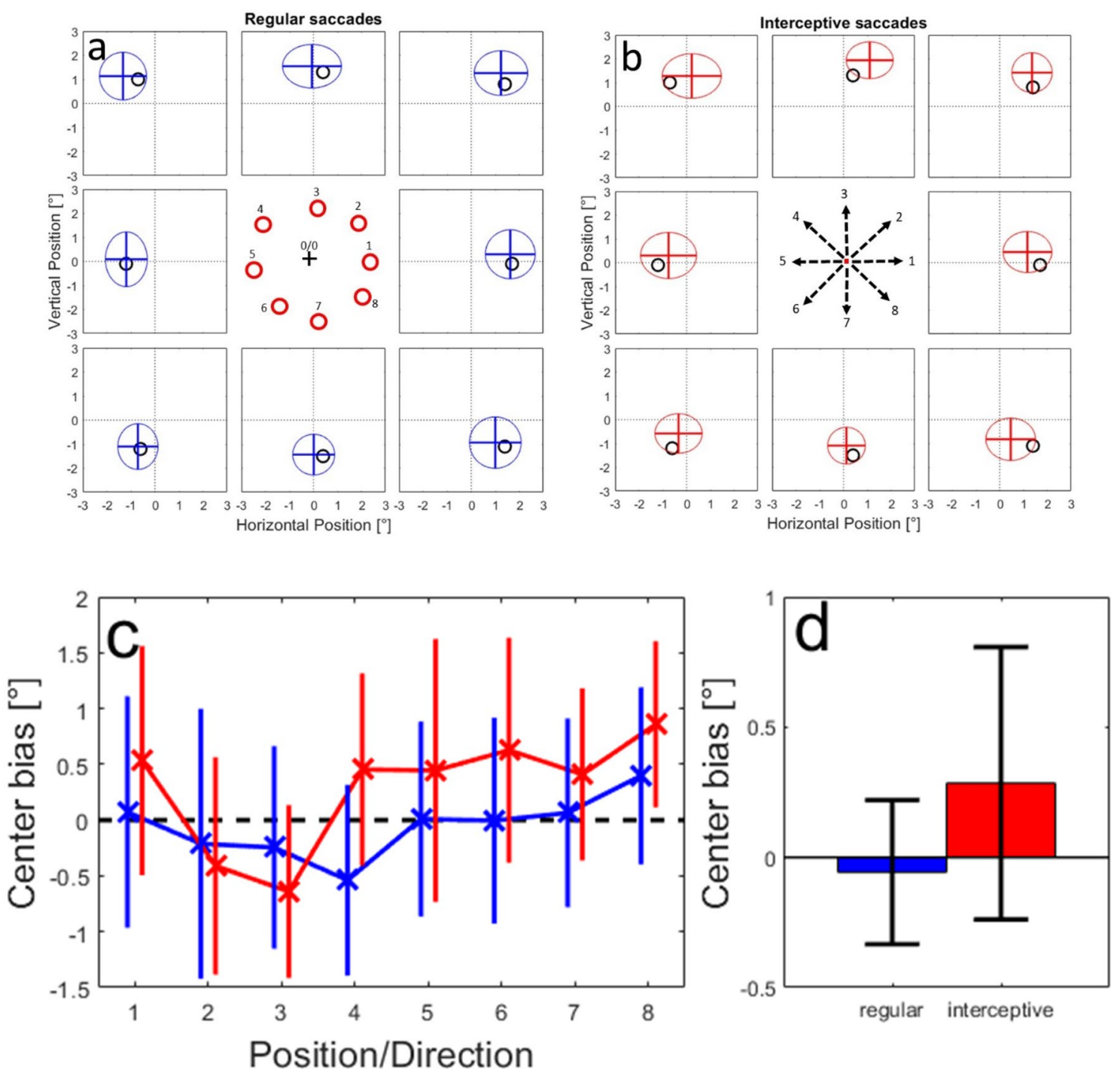

Fig. 10 Same predictions of saccade end-positions as in Fig. 8. Here, the networks were trained based on activity of 25 neurons with the strongest similarity between the activity profiles for regular and interceptive saccades

subjects and tested macaques which may results in different oculomotor performance.

\section{Role of area LIP in generation of interceptive saccades}

Our results together with our earlier study (Bremmer et al. 2016) clearly point towards an involvement of LIP neurons in the representation of interceptive saccades. Our results are clearly inconsistent with the notion that LIP neurons only code for a 'snapshot' of the target position taken just after the initial target step since the motion direction of the target after this step can be successfully recovered from the neuronal activity. Given this principal finding it should be, however, more precisely specified to what degree LIP neurons contribute to the very accurate interception of the target position at the end of the saccade. It is, e.g., possible, that although the neurons do not represent a snapshot of the target position taken immediately after the target step, they still may use a snapshot taken at some later point in time, when the target already moved for a distance in the given direction, and thus partly represent the motion of the target without explicitly processing the motion signal. We believe that this interpretation of our results is unlikely. In our sample of neurons, we found a continuum regarding the representation of the stimulus motion by single neurons. When we predicted the saccade landing position of interceptive saccades based on all investigated neurons, the predicted landing position lagged on average by $0.8^{\circ}$ which corresponds to $80 \mathrm{~ms}$ of target motion, which is consistent with a position snapshot 

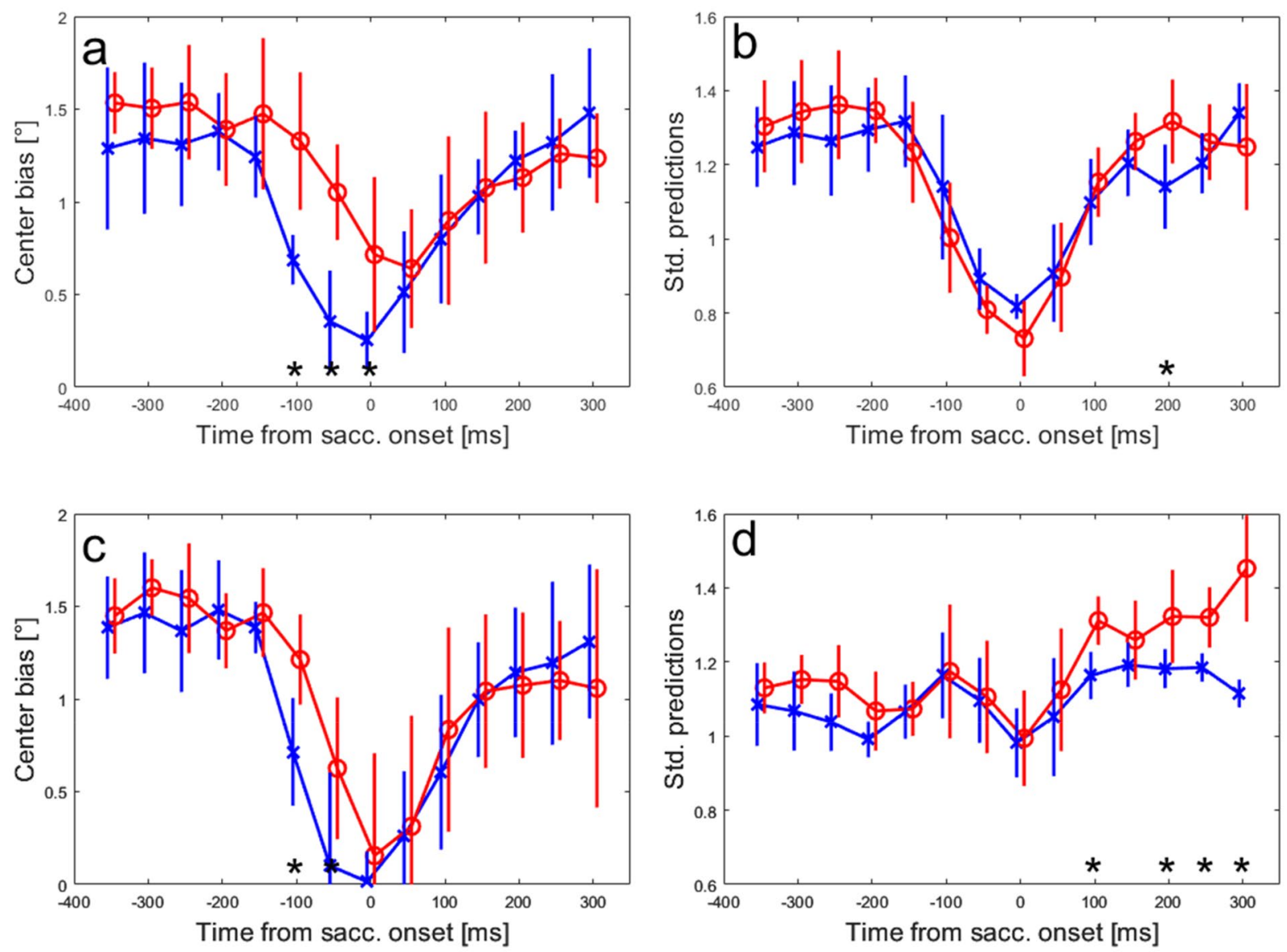

Fig. 11 a Average center bias of predicted end points for regular (blue) and interceptive (red) saccades in different time-windows relative to saccade onset. The $\mathrm{x}$-axes show the center of a $100 \mathrm{~ms}$ time-window from which the results were calculated, the error bars show standard deviations over the eight tested positions. b Average standard deviations of the predicted saccade end-positions for regu-

taken $\sim 50 \mathrm{~ms}$ before the saccade onset. However, when only those $\sim 25 \%$ of neurons with the strongest similarity between the tuning profiles for regular and interceptive saccades, the time was reduced to $30 \mathrm{~ms}$ which is approximately the time of saccade onset. Our model may, however, constitute rather a lower estimate of the actual coding accuracy of interceptive saccades in area LIP. On the one hand, it is not clear whether, given an even more extensive sampling of LIP neurons, another sub-population would have emerged, that would even more accurately represent the target position at the time of saccade end. On the other hand, the differences in sampling positions between the regular and the interceptive saccades that were unavoidable during the measurements may have reduced the similarity of tuning profiles and in that way also the accuracy of the model predictions. Also, the uneven distribution of the preferred directions in our data sample (as shown in Figure S-1) may have resulted in an uneven accuracy of the predictions in different parts of the

lar (blue) and interceptive (red) saccades. c, d Show the same measures as $\mathbf{a}, \mathbf{b}$, only based on the predictions derived from a sub-sample of 25 neurons that have shown the strongest similarity between the activity profiles of regular and interceptive saccades. The asterisks at the bottom of each plot indicate time-windows with significant (paired $t$ test, $p<0.01$ ) differences between the two saccade types

tested area (as observed in Fig. 9b). Nevertheless, the current data allow for decoding of saccades that represent the target position up to the saccade onset, which gives further credibility to the idea that (given the processing latencies from LIP to the motor neurons) the neurons did not operate based just on a snapshot but on a genuine processing of a motion signal (Assad and Maunsell 1995).

Our findings resemble an earlier report from the intermediate and deep layers of the SC (Goffart et al. 2017) that has also shown a continuum in degrees to which neurons changed the position of their motion fields during interceptive saccades. The SC is by far the most investigated area regarding its contributions to the generation of interceptive saccades. An early report (Keller et al. 1996) has shown that the preferred motion vector of saccade-related SC neurons moved in the direction of target motion during interceptive saccades relative to saccades to stationary targets. This finding can be interpreted as a lack of contribution of 
SC neurons to the accurate metrics of interceptive saccades and thus a second drive was postulated that contributes the target-motion-related component of the saccade.

Areas LIP and SC are strongly interconnected (Andersen et al. 1985) so that it is possible that the properties reported by Goffart et al. are inherited from area LIP. Both reports suggest that a second drive that further increases the accuracy of interceptive saccades is likely. It is not clear so far, whether this second drive comes from the activity of the Fastigial nucleus of the cerebellum as it was hypothesized earlier (Optican and Quaia 2002; Optican and Pretegiani 2017) or whether it is contributed by the FEF, where motion information was reported (Barborica and Ferrera 2003). These possibilities are of course not exclusive.

\section{Different approaches for decoding of saccades from neuronal activity}

In this manuscript, we present a population-centered approach to the coding of interceptive saccades. This is slightly different from previous works (e.g., Keller et al. 1996; Goffart et al. 2017) that focused on the accurate description of single neurons their discharging properties and motion fields.

We acknowledge, that other models can be used to decode saccade trajectories from the activity of a neuronal sample. In previous publications (Bremmer et al. 2016; Churan et al. 2019), we used a maximum likelihood approach for the same purpose. In that approach, we generated probability maps of different saccade end-positions based on the activity of each neuron and generated a population prediction by combining all probability maps for the specific activity patterns that were found around the time of the interceptive saccades. In particular, in Churan et al. (2019), this approach was used on partly the same data as in this manuscript. Despite the large methodological differences, the basic conclusions derived from these two approaches were very similar. Our current approach, however, allowed more clear-cut results that appeared more stable (e.g., across different neuronal samples) and independent of outliers than they were when the maximum likelihood approach was used.

\section{Future directions}

As mentioned above, our findings point in the same direction as previous results from the superior colliculus (Goffart et al. 2017). The methods chosen in that study were different from ours and thus it would be intriguing to use our decoding approach on a sample of SC neurons to investigate whether the coding accuracy for regular and interceptive saccades is similar in SC.
A recent development has shown a promising way to further disentangle the contributions of target position and target motion for generation of interceptive saccades. Goettker et al. (2019) have demonstrated that when human subjects were asked to perform interceptive saccades towards isoluminant targets, the interceptive saccades become inaccurate and lag behind the moving target by $\sim 100 \mathrm{~ms}$. This is attributed to a lack of motion information provided by isoluminant stimuli (Cavanagh et al. 1984; Lu et al. 1999) to areas of the dorsal processing stream like the middle temporal, and middle superior temporal areas (Thiele et al. 1999; Riečanský et al. 2005). Thus, it would be intriguing to investigate how the processing of interceptive saccades in LIP and SC changes between luminance defined and isoluminant stimuli.

Supplementary Information The online version contains supplementary material available at https://doi.org/10.1007/s00429-021-02365-x.

Acknowledgements This work was supported by Deutsche Forschungsgemeinschaft (CRC/TRR-135/A1 [project number 222641018], IRTG-1901, and RU 1847/A2) and by the Hessisches Ministerium für Wissenschaft und Kunst (HMWK; project 'The Adaptive Mind').

Funding Open Access funding enabled and organized by Projekt DEAL. This work was supported by Deutsche Forschungsgemeinschaft (CRC/TRR-135/A1 [project number 222641018], IRTG-1901, and RU 1847/A2) and by the Hessisches Ministerium für Wissenschaft und Kunst (HMWK; project 'The Adaptive Mind').

Availability of data and code The datasets generated during and/or analyzed during the current study as well as the code used for evaluating the data are available from the corresponding author on reasonable request.

\section{Declarations}

Conflict of interest The authors have no conflicts of interest to declare that are relevant to the content of this article.

Ethics approval All procedures had been approved by the regional authorities (TVA Nr.: V54-19 c 2015 h 01 MR 13/1 Nr. G71/2017) and were in accordance with the published guidelines on the use of animals in research (European Communities Council Directive 2010/63/EU).

Open Access This article is licensed under a Creative Commons Attribution 4.0 International License, which permits use, sharing, adaptation, distribution and reproduction in any medium or format, as long as you give appropriate credit to the original author(s) and the source, provide a link to the Creative Commons licence, and indicate if changes were made. The images or other third party material in this article are included in the article's Creative Commons licence, unless indicated otherwise in a credit line to the material. If material is not included in the article's Creative Commons licence and your intended use is not permitted by statutory regulation or exceeds the permitted use, you will need to obtain permission directly from the copyright holder. To view a copy of this licence, visit http://creativecommons.org/licenses/by/4.0/. 


\section{References}

Andersen RA, Asanuma C, Cowan WM (1985) Callosal and prefrontal associational projecting cell populations in area 7A of the macaque monkey: a study using retrogradely transported fluorescent dyes. J Comp Neurol 232(4):443-455. https://doi. org/10.1002/cne.902320403

Assad JA, Maunsell JHR (1995) Neuronal correlates of inferred motion in primate posterior parietal cortex. Nature 373(6514):518-521. https://doi.org/10.1038/373518a0

Barash S, Bracewell RM, Fogassi L, Gnadt JW, Andersen RA (1991) Saccade-related activity in the lateral intraparietal area. I. Temporal properties; comparison with area 7a. J Neurophysiol 66(3):1095-1108. http://www.ncbi.nlm.nih.gov/pubmed/17532 76

Barborica A, Ferrera VP (2003) Estimating invisible target speed from neuronal activity in monkey Frontal eye field. Nat Neurosci 6(1):66-74. https://doi.org/10.1038/nn990

Blatt GJ, Andersen RA, Stoner GR (1990) Visual receptive field organization and cortico-cortical connections of the lateral intraparietal area (area LIP) in the macaque. J Comp Neurol 299(4):421-445. https://doi.org/10.1002/cne.902990404

Brainard DH (1997) The Psychophysics Toolbox. Spat Vis 10(4):433436. http://color.psych.ucsb.edu/psychtoolbox

Bremmer F, Kaminiarz A, Klingenhoefer S, Churan J (2016) Decoding target distance and saccade amplitude from population activity in the macaque lateral intraparietal area (LIP). Front Integr Neurosci. https://doi.org/10.3389/fnint.2016.00030

Cassanello CR, Nihalani AT, Ferrera VP (2008) Neuronal responses to moving targets in monkey frontal eye fields. J Neurophysiol 100(3):1544-1556. https://doi.org/10.1152/jn.01401.2007

Cavanagh P, Tyler CW, Favreau OE (1984) Perceived velocity of moving chromatic gratings. J Opt Soc Am A 1(8):893. https://doi.org/ 10.1364/josaa.1.000893

Churan J, Braun DI, Gegenfurtner KR, Bremmer F (2018) Comparison of the precision of smooth pursuit in humans and head unrestrained monkeys. J Eye Mov Res 11(4):1-15. https://doi.org/10. 16910/JEMR.11.4.6

Churan J, Braun DI, Gegenfurtner KR, Kaminiarz A, Bremmer F (2019) Encoding of interceptive saccades in parietal cortex of macaque monkeys. Program No. 144.07. 2019 Neuroscience Meeting Planner. Society for Neuroscience, Chicago, IL

Ferrera VP, Barborica A (2010) Internally generated error signals in monkey frontal eye field during an inferred motion task. J Neurosci 30(35):11612-11623. https://doi.org/10.1523/JNEUROSCI. 2977-10.2010

Fleuriet J, Goffart L (2012) Saccadic interception of a moving visual target after a spatiotemporal perturbation. J Neurosci 32(2):452461. https://doi.org/10.1523/JNEUROSCI.3896-11.2012

Fuchs AF (1967) Saccadic and smooth pursuit eye movements in the monkey. J Physiol 191(3):609-631. https://doi.org/10.1113/jphys iol.1967.sp008271

Gellman RS, Carl JR (1991) Motion processing for saccadic eye movements in humans. Exp Brain Res 84(3):660-667. https://doi.org/ 10.1007/BF00230979

Goettker A, Braun DI, Gegenfurtner KR (2019) Dynamic combination of position and motion information when tracking moving targets. J vis. https://doi.org/10.1167/19.7.2

Goffart L, Cecala AL, Gandhi NJ (2017) The superior colliculus and the steering of saccades toward a moving visual target. J Neurophysiol 118(5):2890-2901. https://doi.org/10.1152/jn.00506.2017

Guan Y, Eggert T, Bayer O, Büttner U (2005) Saccades to stationary and moving targets differ in the monkey. Exp Brain Res 161(2):220-232. https://doi.org/10.1007/s00221-004-2070-3
Keller EL, Gandhi NJ, Weir PT (1996) Discharge of superior collicular neurons during saccades made to moving targets. J Neurophysiol 76(5):3573-3577. https://doi.org/10.1152/jn.1996.76.5.3573

Kleiner M, Brainard D, Pelli D, Ingling A, Murray R, Broussard C (2007) What's new in psychtoolbox-3. Perception 36(14):1-16

Levenberg K (1944) A method for the solution of certain non-linear problems in least squares. Q Appl Math 2(2):164-168. https:// doi.org/10.1090/qam/10666

Lu ZL, Lesmes LA, Sperling G (1999) Perceptual motion standstill in rapidly moving chromatic displays. Proc Natl Acad Sci USA 96(26):15374-15379. https://doi.org/10.1073/pnas.96.26.15374

Marquardt DW (1963) An algorithm for least-squares estimation of nonlinear parameters. J Soc Ind Appl Math 11(2):431-441. https://doi.org/10.1137/0111030

Newsome WT, Wurtz RH, Dursteler MR, Mikami A (1985) Deficits in visual motion processing following ibotenic acid lesions of the middle temporal visual area of the macaque monkey. J Neurosci 5(3):825-840. https://doi.org/10.1523/jneurosci.0503-00825.1985

Optican LM, Pretegiani E (2017) What stops a saccade? Philos Trans R Soc B. https://doi.org/10.1098/rstb.2016.0194

Optican LM, Quaia C (2002) Distributed model of collicular and cerebellar function during saccades. Ann N Y Acad Sci 956(1):164-177. https://doi.org/10.1111/j.1749-6632.2002. tb02817.x

Paré M, Wurtz RH (1997) Monkey posterior parietal cortex neurons antidromically activated from superior colliculus. J Neurophysiol 78(6):3493-3497. https://doi.org/10.1152/jn.1997.78.6.3493

Pelli DG (1997) The VideoToolbox software for visual psychophysics: transforming numbers into movies. Spat Vis 10(4):437-442. http://www.ncbi.nlm.nih.gov/pubmed/9176953

Plexon (2020) Offline Sorter: user guide. https://plexon.com/wp-conte nt/uploads/2020/01/Offline-Sorter-v4-User-Guide.pdf

Quinet J, Goffart L (2015) Does the brain extrapolate the position of a transient moving target? J Neurosci 35(34):11780-11790. https:// doi.org/10.1523/JNEUROSCI.1212-15.2015

Riečanský I, Thiele A, Distler C, Hoffmann KP (2005) Chromatic sensitivity of neurones in area MT of the anaesthetised macaque monkey compared to human motion perception. Exp Brain Res 167(4):504-525. https://doi.org/10.1007/s00221-005-0058-2

Schall JD, Morel A, King DJ, Bullier J (1995) Topography of visual cortex connections with frontal eye field in macaque: convergence and segregation of processing streams. J Neurosci 15(6):44644487. https://doi.org/10.1523/jneurosci.15-06-04464.1995

Schiller PH, True SD, Conway JL (1980) Deficits in eye movements following frontal eye-field and superior colliculus ablations. J Neurophysiol 44(6):1175-1189. https://doi.org/10.1152/jn.1980. 44.6.1175

Segraves MA, Goldberg ME (1987) Functional properties of corticotectal neurons in the monkey's frontal eye field. J Neurophysiol 58(6):1387-1419. https://doi.org/10.1152/jn.1987.58.6.1387

Shadlen MN, Newsome WT (1998) The variable discharge of cortical neurons: implications for connectivity, computation, and information coding. J Neurosci 18(10):3870-3896. https://doi.org/10. 1523/jneurosci.18-10-03870.1998

Sotero RC, Bortel A, Martínez-Cancino R, Neupane S, O'Connor P, Carbonell F, Shmuel A (2010) Anatomically-constrained effective connectivity among layers in a cortical column modeled and estimated from local field potentials. J Integr Neurosci 9(4):355-379. https://doi.org/10.1142/S0219635210002548

Stanton GB, Goldberg ME, Bruce CJ (1988) Frontal eye field efferents in the macaque monkey: II. Topography of terminal fields in midbrain and pons. J Comp Neurol 271(4):493-506. https://doi.org/ $10.1002 /$ cne. 902710403 
Thiele A, Dobkins KR, Albright TD (1999) The contribution of color to motion processing in macaque middle temporal area. J Neurosci 19(15):6571-6587. https://doi.org/10.1523/jneurosci.19-1506571.1999

Xiao Q, Barborica A, Ferrera VP (2007) Modulation of visual responses in macaque frontal eye field during covert tracking of invisible targets. Cereb Cortex 17(4):918-928. https://doi.org/10. 1093/cercor/bhl002

Publisher's Note Springer Nature remains neutral with regard to jurisdictional claims in published maps and institutional affiliations. 